\title{
Is the CPAP estimate of the apnea-hypopnea index on therapy clinically useful?
}

\author{
M. Jeffery Mador
}

Received: 9 February 2012 /Revised: 9 February 2012 / Accepted: 10 February 2012 /Published online: 6 March 2012

(C) Springer-Verlag 2012

Sleep apnea is extremely common in middle-aged adults [1]. The traditional model of evaluating sleep apnea has been to perform a diagnostic in-lab study to diagnose sleep apnea and then an in-lab CPAP titration study to determine the optimal CPAP pressure to treat sleep apnea. This approach is costly and labor intensive. There is considerable interest in adopting alternative treatment algorithms that would reduce the need for in-lab sleep studies. In most countries, the demand for sleep studies exceeds capacity. Auto-CPAP devices have been developed that adjust the pressure delivered to meet the patient's requirements during sleep. If these devices were completely effective, there would be no need for an in-lab titration as the patient could either continue on auto-CPAP or the fixed pressure could be determined from review of the pressures delivered during auto-CPAP. CPAP devices now provide reports where they estimate the apneahypopnea index (AHI) on therapy. There is a relative paucity of data addressing the accuracy of these AHI estimates.

In this issue of Sleep and Breathing, Cilli and colleagues compare the AHI estimated by the CPAP machine with that obtained during formal polysomnography while the patient is on auto-CPAP. They found that auto-CPAP was successful in treating sleep apnea in the vast majority of patients. Only 2 out of $137(1.5 \%)$ had an AHI greater than 10/h on therapy. Furthermore, the CPAP AHI was similar to the AHI measured in lab with limits of agreement of +4.9 to $-4.8 / \mathrm{h}$. Thus, it would appear at first glance that auto-CPAP is quite effective at alleviating sleep apnea and that the CPAP estimate of AHI

M. J. Mador $(\bowtie)$

Western New York Veteran Affairs Healthcare System and Division of Pulmonary, Critical Care and Sleep Medicine,

State University of New York at Buffalo,

Section 111S, 3495 Bailey Avenue,

Buffalo, NY 14215, USA

e-mail: mador@buffalo.edu was quite accurate. Let us examine these two conclusions in greater detail.

Two recent studies have also examined the efficacy of auto-CPAP at alleviating sleep apnea; in the first, a residual AHI on auto-CPAP greater than $5 / \mathrm{h}$ was seen in $26 \%$ of patients and greater than $10 / \mathrm{h}$ in $17 \%$ of patients [2]. In another smaller study using a different CPAP device, $24 \%$ of patients had a residual AHI greater than $10 / \mathrm{h}$ on auto-CPAP [3]. Thus, auto-CPAP is not always successful at alleviating sleep-disordered breathing. Perhaps surprisingly, a similar proportion of patients (17\%) had residual sleep apnea (defined as an AHI greater than 10/h) after in-lab CPAP titration which is felt to be the gold standard [4]. These patients generally did not have any symptoms suggesting persistence of sleep apnea. Thus, suboptimal control of sleep apnea is not uncommon after treatment with auto-CPAP and even after in-lab titration. If an increased AHI on therapy in the absence of symptoms is unhealthy for the patient by for example increasing cardiovascular risk, then detection would be highly useful. The CPAP estimate of AHI on therapy could provide a method for detection of this situation.

How accurate is this CPAP estimate at detecting a residually increased AHI on therapy? Unfortunately, the present study cannot really address this important issue since only two patients had an AHI above 10/h on therapy. Similar to a prior study [2] using a similar CPAP machine, the CPAP estimate of AHI tended to overestimate the AHI at lower AHIs and underestimate the AHI at higher AHIs. In this prior study using an arbitrary cutoff of 10/h, 13 out of the 17 patients who had an in-lab AHI greater than $10 / \mathrm{h}$ also had a CPAP AHI estimate over $10 / \mathrm{h}$. Taking into account the underestimation of AHI at higher values by the CPAP machine, a CPAP AHI of less than $8 / \mathrm{h}$ was rarely associated with an in-lab AHI of greater than 10/h. In other studies using a different CPAP device, the device also tended to 
overestimate the AHI at lower values and underestimate it at higher values [3] or consistently overestimated the AHI [5]. Detection of a residually increased AHI on therapy was worse with only three out of eight patients detected [3].

Another potential problem with the CPAP estimate of AHI is that it could falsely suggest an increased AHI on therapy leading to unnecessary further investigation. Perusal of Fig. 1 in this study suggests that this was relatively uncommon. A prior study using a similar device found an increased CPAP AHI that was not substantiated in lab in 3 out of 82 patients (3.7\%) [2]. Another study using a different CPAP device found a falsely elevated CPAP AHI in 5 out of 26 patients (19.2\%) [3].

Each CPAP machine has its own internal algorithms and the results with one CPAP device cannot be extrapolated to another as shown above. Another potential concern is that CPAP manufacturers can tweak their internal algorithms in an effort to make them better but this may make prior evaluations using an older software iteration unreliable.

In summary, it is not uncommon for the AHI to be residually elevated on auto-CPAP or even fixed pressure CPAP. Obviously, if the patient has persistent symptoms that are unexplained by suboptimal compliance or alternative sleep disorders, further investigation is indicated regardless of the
CPAP estimate of AHI. In the absence of symptoms, the CPAP estimate of AHI provides an important clue that CPAP therapy may not be truly optimal but the clinician must be aware of its limitations.

\section{References}

1. Young T, Palta M, Dempsey J, Skatrud J, Weber S, Badr S (1993) The occurrence of sleep-disordered breathing among middle-aged adults. N Engl J Med 328(17):1230-1235

2. Desai H, Patel A, Patel P, Grant BJB, Mador MJ (2009) Accuracy of autotitrating CPAP to estimate the residual apnea-hypopnea index in patients with obstructive sleep apnea on treatment with autotitrating CPAP. Sleep Breath 13:383-390

3. Denotti AL, Wong KH, Dungan GC, Gilholme JW, Marshall NS, Grunstein RR (2012) Residual sleep disordered breathing during auto-titrating positive pressure therapy. Eur Respir J. doi:10.1183/ 09031936.00093811

4. Baltzan MA, Kassissia I, Elkholi O, Palayew M, Dabrusin R, Wolkove N (2006) Prevalence of persistent sleep apnea in patients treated with continuous positive airway pressure. Sleep 29:557-563

5. Ueno K, Kasai T, Brewer G, Takaya H, Maeno K-I, Kasagi S, Kawana F, Ishiwata S, Narui K (2010) Evaluation of the apneahypopnea index determined by the S8 auto-CPAP, a continuous positive airway pressure device, in patients with obstructive sleep apnea-hypopnea syndrome. J Clin Sleep Med 6:146-151 